\title{
Single formant contrast in vowel identification
}

\author{
ROBERT G. CROWDER \\ Yale University and Haskins Laboratories, New Haven, Connecticut
}

and

\author{
BRUNO H. REPP \\ Haskins Laboratories, New Haven, Connecticut
}

\begin{abstract}
Subjects rated ambiguous steady-state vowels from a continuum with respect to the categories /i/ and /I/ (Experiment 1) or / $/$ / and / a/ (Experiment 2). Each target was preceded, .35 sec earlier, by one of the following precursors: (1) one endpoint from the target continuum, (2) the other endpoint, (3) the isolated first formant (F1) from (1), (4) the isolated F1 from (2), or (5), a hissing noise. Although (3) and (4) did not sound like they came from the target continuum, they produced reliable contrast in both experiments. In the $/ \mathrm{i} / \mathrm{-} / \mathrm{I} /$ experiment, contrast was as powerful from single formants as from the full vowels. These results suggest a sensory, rather than a judgmental, basis for the vowel contrast effects obtained.
\end{abstract}

The occurrence of contrast in perceptual judgments along single dimensions is so commonplace it seems almost uninteresting. In judging shades of gray, heaviness of lifted objects, line lengths, loudness of tones, and so on, the perceived magnitude of one stimulus is usually affected contrastively by that of another stimulus with which it is presented. A patch of gray seems dark against a white background, and yet the same patch seems light against a black background, for example. The pervasiveness of contrastive interactions between nearby stimuli should not, however, lead us to forget how important it is. Contrast allows the perceptual system to focus on what otherwise might be elusive differences. It hardly requires discussion that edge-sharpening, in vision, advances the more informative aspects of the visual world at the expense of the less informative aspects.

The example of visual brightness contrast is an interesting one because a detailed neurophysiological basis for it has been worked out (however, see Gilchrist, 1977). Edge sharpening in at least simultaneous brightness contrast follows inescapably from verified rules of recurrent lateral inhibition in the visual (retinal) system (see summary in Lindsay \& Norman, 1977). Where does this leave us with other

Part of the preparation of this manuscript was done while R. G. Crowder was a fellow at the Center for Advanced Study in the Behavioral Sciences, Stanford, CA. We are grateful to the following sources of support: NSF Grant BNS 8206304 to the Center for Advanced Study in the Behavioral Sciences, NICHD Grant HD-01994 and BRS Grant RR-05596 to Haskins Laboratories, and NSF Grant BNS 8005838 to R. G. Crowder. We appreciate the able assistance of Virginia Walters and Herta Flor in collecting and analyzing the data. R. G. Crowder's mailing address is: Psychology Department, Box 11a, Yale Station, New Haven, CT 06520. kinds of contrast, though? It would be grandiose to apply the neural circuitry proposed for brightness contrast to, say, contrast effects in judging the conservatism of Supreme Court Justices. Some kinds of contrast, in other words, might be more "cognitive" or judgmental and others more sensory. (This comment need not seem like an eruption of the mind/ body distinction: Infants would display the sensory forms of contrast but not the judgmental forms.)

In fluent speech perception, most especially in unstressed, "reduced" vowels, the stimulus information is often impoverished relative to prototypical category instances. In a speaker's haste to get from one to another consonantal gesture, he often "misses" producing a vowel sound in anything like its citation form. Veridical perception would be well served, in these cases, by a process of "edge sharpening" for the vowel perception system, so that surviving acoustic stimulus distinctions would be exaggerated. Indeed, since the work of Fry, Abramson, Eimas, and Liberman (1962), it has been known that isolated vowels show contrastive context effects in identification judgments. Sawusch, Nusbaum, and Schwab (1980) have distinguished three classes of explanation for the various instances of vowel contrast that have been reported in the intervening years. They discount feature detector fatigue as an explanation because there are often substantial time lapses between context and target. We can also mention that retroactive contrast (Diehl, Elman, \& McCusker, 1978; Repp, Healy, \& Crowder, 1979) in which the target comes first and the context second-effectively dismisses this first explanation. Changes in auditory ground and response bias are the two remaining classes of hypothesis. Sawusch et al. (1980) apply these inter- 
pretations mainly to contrast elicited in an anchoring paradigm and we need not follow these applications here in detail. It suffices to remark that the auditoryground interpretation appeals to sensory contrast in a way that is congenial with the analogy to visual brightness contrast, whereas response bias would very clearly be a judgmental process.

Some recent research on selective adaptation in the perception of stop consonants has pointed towards auditory-sensory explanations rather than towards judgmental explanations. In the selective adaptation paradigm, repeated presentations of an adaptor stimulus are shown to affect the perception of a subsequent test stimulus. The experimental operations involved in measuring selective adaptation are obviously a special case of contrast, several authors having suggested a profound continuity of process between the two (Crowder, 1981; Diehl et al., 1978; Diehl, Lang, \& Parker, 1980). In two such experiments, the authors were able to pit sensory (spectral) and judgmental factors against each other. In one of these studies, Roberts and Summerfield (1981) used an audio-visual adaptor in which an acoustic /be/ was synchronized with a visual $/ \mathrm{ge} /$. The combination was identified as $/ \mathrm{de} /$ or $/ \delta \mathrm{e} /$; however, its effect on perception of a /be-de/ test series was identical to that of an unambiguous acoustic /be/. Thus, perception responded to the spectral, and not the perceived phonetic, nature of the adaptor. In the other experiment (Sawusch \& Jusczyk, 1981), an adaptor was made from a fricative-stop-vowel syllable $/ \mathrm{s}+\mathrm{ba} /$ with $75 \mathrm{msec}$ of silence between the two segments. Under these conditions, subjects call the adaptor syllable "spa" even though the stop-vowel portion, alone, is unambiguously / $\mathrm{ba} /$. In a /ba-pa/ test series following these and other adaptors, the "perceptual /spa/" but "acoustic $/ \mathrm{s}+\mathrm{ba} /$ " affected responses just the same way as did an unambiguous /ba/, again showing the spectral cue to prevail even in the face of contradictory labeling.

In their experimental work with isolated vowels from the $/ \mathrm{i} /-/ \mathrm{l} / \mathrm{continuum}$, Sawusch et al. (1980) reached the interesting conclusion that both sensory and judgmental factors contribute to contrast, but at different ends of the continuum. Their technique involved measurement of discrimination sensitivity as well as identification ratings. When / $i /$ was the context (anchor), there seemed to be genuine changes in the sharpness of the sensory system in the / $i$ / range of the continuum. When /I/ was the context, however, the changes seemed rather to be in where people placed their response criterion. Thus, within the same stimulus continuum, we may be able to see more than one form of contrast operating.

\section{EXPERIMENT 1}

The present report extends the Sawusch et al. work in several ways, but it explores the same question of where contrast effects should be located with regard to the sensory versus response end of the processing machinery. In our experiment, pairs of isolated vowel sounds were presented in rapid $(.35-\mathrm{sec})$ succession. The stimuli all came from a seven-item /i/-/I/ continuum, varying only in F1 frequency. (The stimuli used by Sawusch et al. varied in F2 as well as F1.) The second item in each pair was the target (second through sixth item from the continuum), and the first was either of the two endpoint items, either of these two items with F2 and F3 removed, or a control (hiss). No response was required to the first item in each pair, the context item. These conditions were either mixed together randomly in a continuous series of trials or were presented in blocks. In the latter arrangement, we should be in a position to observe the anchoring effects found by Sawusch et al. as well as "regular" contrast between the two items in a pair. When the conditions are randomized, however, only pairwise contrast should be observed. Our choice of the neutral hiss in the control condition was considered: We wanted as close to a "no-contrast" condition as we could get. Any tone or vowel, however unrelated to the test continuum it seemed, carried potential spectral or phonetic bias. The hiss served as a simple warning signal with no such bias.

Because removal of F2 and F3 results in these items' sounding unlike tokens of the $/ \mathrm{i} /-/ \mathrm{I} /$ continuum, we can also offer expectations for which contrast effects ought to be influenced by whether the precursors are intact three-formant vowels or not. If Sawusch et al. are correct in assigning contrast produced by $/ i /$ to sensory factors, we might expect that removal of F2 and F3 would make little difference. For example, Crowder $(1981,1982)$ has proposed a theory of frequency-specific recurrent lateral inhibition (see below) that anticipates the same degree of contrast whether or not F2 and F3 are present. If contrast from the /I/ side of the continuum is produced by other factors, perhaps response bias or a rangefrequency effect (Parducci, 1974), then removal of F2 and F3 might alter the situation, because the tacit labels that subjects might assign to the precursors (and which might engage the judgmental bias) would be foreign to the target $/ \mathrm{i} /-/ \mathrm{I} /$ continuum. Furthermore, if a sort of adaptation-level mechanism contributed to contrast in the anchoring situation (Sawusch et al., 1980), then we should expect more contrast in the blocked arrangement of conditions than in the randomized arrangement; this is because in the blocked arrangement the same single precursor is the first item in each pair and therefore vastly outnumbers each of the six items that can be the second item in the pair.

\footnotetext{
Method

Subjects. The subjects were 20 Yale undergraduates, serving either for pay or for course credit.

Stimuli. The basic continuum of seven vowels was prepared on the Haskins Laboratory parallel resonance synthesizer. The
} 
items were designed to range perceptually from /i/ to /I/ and varied only in F1 center frequencies (from 279 to $381 \mathrm{~Hz}$ in roughly equal steps). F2 and F3 center frequencies were kept fixed at 2075 and $2780 \mathrm{~Hz}$, respectively. These frequencies were compromise values between those typical of the vowels / $i$ / and /I/ (Peterson \& Barney, 1952). Three additional stimuli were used, (1) the /i/ endpoint from the continuum with F2 and F3 removed (through options within the synthesizer), (2) the / $/$ endpoint modified in the same way, and (3) a soft hiss, which served as a control. All stimuli were $300 \mathrm{msec}$ long. The vowels rose in fundamental frequency from 80 to $100 \mathrm{~Hz}$ during the first $100 \mathrm{msec}$ and declined to $85 \mathrm{~Hz}$ during the last $100 \mathrm{msec}$. The amplitude envelope was likewise shaped at the beginning and end of the syllable. In the stimuli with F2 and F3 removed, the amplitude of F1 matched F1 amplitude in the corresponding full vowels. However, the overall amplitude was reduced by removal of F2 and F3.

In a preliminary experiment, 25 subjects were given single-item identification tests on vowels similar to the vowels lacking F2 and F3 used in the present experiment. Other details of that preliminary experiment need not concern us: It included contrast comparisons similar to, but superceded by, those of the present experiment. Nothing in the preliminary study compromises, however, what we found later. Of interest now is that these 25 subjects were asked to listen to tokens of the various precursors in isolation and report what they sounded like, with examples of words containing the sounds. It has long been known that people can perceive and classify single-formant vowels, and that low-frequency single formants are heard as back vowels (Delattre, Liberman, Cooper, \& Gerstman, 1952). When describing the /i/ endpoint of the vowels described above, but with F2 and F3 deleted, 24 of the 25 subjects reported that it sounded like / $u$ / (BOOT) and the remaining subject reported $/ \ni /$. Given the $/ 1 /$ endpoint, 7 responded with the same vowel $(/ \mathrm{u} /), 14$ with the sound /o/ (BOAT), 3 with $/ N$, and 1 with $/ \partial /$, but never $/ I /$. Thus, we may be assured that removal of the second and third formants did indeed drive labeling away from the $/ \mathrm{i} /-/ \mathrm{I} /$ continuum.

The experimental tapes contained 100 trials (pairs of vowels) apiece. Each trial included the precursor, a 350-msec delay, and then the target; after the offset of the target, there was a 2.5-sec delay before the beginning of the next trial. After every 10 trials, there was a longer intertrial delay $(5 \mathrm{sec})$ intended to help subjects keep their places.

Procedure. Subjects in the blocked condition received five 100 trial tapes, one for each of the five precursor conditions, in different orders, as determined by a Latin square. The 100 trials with a given precursor included 20 with each of the target vowels (numbers 2 through 6 on the original continuum). Subjects in the random condition heard precisely the same 500 trials, also in batches of 100 ; however, the trials were completely randomized, so that two adjacent trials usually had different precursors.

In the first part of the session, the subjects were played the / $\mathrm{i}$ /I/ continuum three times, in order, from number 2 through number 6 of the original seven. They were told that the first item in the group was "what we are calling EE" and the last, "IH." They all then listened to the first 10 trials on the random tape as practice, and then began the experiment proper. Answer sheets had arabic numbers from 1 to 5 opposite each trial number. Over the left column (the 1s), the word BEET was spelled and over the right column (the 5s), BIT. The procedure was to rate the similarity of each target to the vowels in these two prototypes by circling one of the five numerals.

\section{Results}

The main results are shown in Figure 1, in terms of mean rating in the "IH" direction. The left panel shows ratings when the precursors were the fullvowel endpoints from the continuum; the right panel shows what happened when F2 and F3 were removed

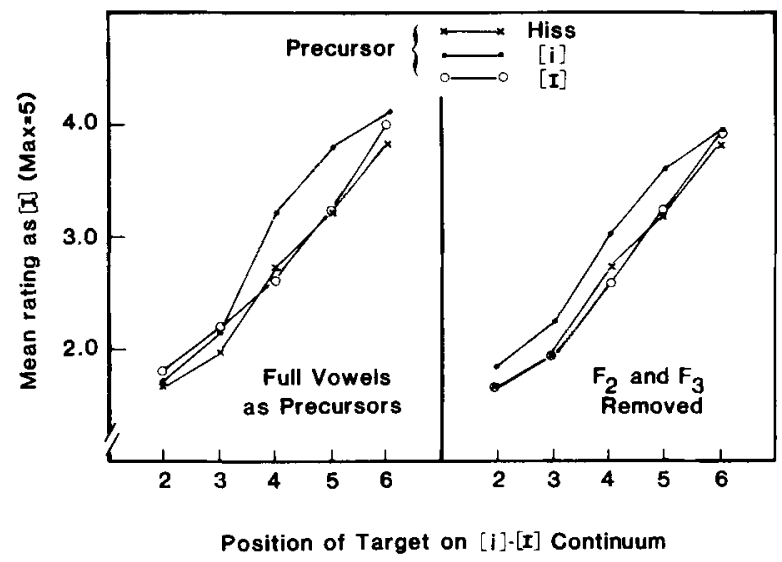

Figure 1. The effect of five context precursors on the relationship between the position of a target vowel along the / $\mathrm{i}-\mathrm{I} / \mathrm{con}$ tinuum and the tendency to rate it as $/ \mathrm{L} /$. The same control condition with a hiss as context is plotted in both panels. On the left, data are shown when the precursor was one or the other of the two series endpoints $/ /$ or $/ I /$. On the right are the results when the same precursors were used with all but the first formant deleted (Experiment 1).

from these stimuli. The hiss condition is drawn in both panels as a baseline.

First of all, there was no result of blocking versus randomizing the experimental conditions, and so Figure 1 combines these two conditions. In a 2 (blocked/ random) $\times 5$ (precursor) $\times 5$ (target position on the continuum) analysis of variance, blocking did not approach the .10 alpha level, either as a main effect or in interactions. However, the same analysis of variance showed that there were reliable differences among the five precursor conditions $[F(4,72)=7.11$, $p<.01]$, differences which interacted with target position $[F(16,288)=3.41, p<.01]$. As the figure shows, precursors had little or no effect on the relatively unambiguous targets-numbers 2 and 6 from the continuum. Of course, the position on the continuum of the target itself had a reliable main effect on ratings of "IH-ness," $[F(4,72)=120.06, p<.01]$.

Contrast was asymmetrical from the two ends of the vowel continuum, to say the least: There was none when /I/ or its single-formant version were used as precursors. However, the $/ \mathrm{i} /$ precursor quite obviously made the targets sound more like /I/, and this was true with or without F2 and F3. The next question is whether the overall degree of contrast was different for the full vowels (left panel) versus the vowels with F2 and F3 removed (right panel). The best single measure of the contrast effect is probably the difference between the / $\mathrm{i} /$ and /I/ precursors (or their modified versions). In a new analysis of variance, the control condition was dropped, and the factors were (1) blocked/random, (2) full/altered precursors, (3) / $i$ / versus /I/ precursor, and (4) position of the target on the continuum. There was a re- 
liable main effect of whether the precursors were altered or not $[F(1,18)=5.25, p<.05]$, reflecting the fact that the full vowel precursors (left panel of Figure 1) led to somewhat higher $/ \mathrm{I} /$ ratings whatever the identity of the precursor $(/ \mathrm{i} /$ or $/ \mathrm{I} /)$. However, the identity of the precursor had a large main effect $[\mathrm{F}(1,18)=13.91, \mathrm{p}<.01]$, and it did not interact with whether the precursors were full or altered $(F<1)$. Thus, there was no evidence that full vowels exert a greater contrast effect than vowels with F2 and F3 removed. There was a statistically significant interaction between these two factors and the position of the target along the continuum $[F(4,72)=3.23, p<$ $.05]$. This interaction reflects the fact that the full vowels had their effects exclusively on the fourth and fifth continuum positions whereas the contrast produced by altered vowels occurred at all continuum positions except the last.

\section{Discussion}

The main finding of the experiment is that contrast was obtained even after F2 and F3 were removed from the endpoint vowels, rendering them phonetically foreign to the continuum being judged. The degree of contrast was not even changed by this operation. It is true that the contrast effect "bulged" differently with the full vowels than with the altered vowels, as revealed by the significant three-way interaction identified in the previous paragraph. We defer comment on this finding until after the second experiment.

Another finding was the asymmetry in contrast across the /i/-/I/ continuum. Although Sawusch et al. (1980) observed anchor effects from both ends of this continuum, and later were able to assign them to different mechanisms, we simply got no contrast at all from $/ 1 /$. At the very least, the asymmetry of this continuum in contrast tells us there is more at work here than a simple judgmental bias leading people to assign contrasting labels to precursor and target. Such a bias would result in symmetrical effects. In fact, if we accept the Sawusch et al. analysis, our results suggest that judgmental bias (associated with the /I/ context) simply did not occur in our experiment.

It made no difference whether conditions were blocked or mixed randomly across trials. This is comforting in that it means there is one less choice to worry about in designing experiments. It was disappointing in the context of this experiment, however, for if contrast from /I/ precursors were a consequence of judgmental bias, one might have expected blocking to make a difference, and differentially for the full and the altered vowels. One possibility is that judgmental bias was not engaged in the pairwise, precursor-target trial arrangement because the precursor never required an overt labeling response. In the anchoring literature, all items are iden- tified in sequence. Perhaps requiring people to label both of the two items in each pair (as in Repp et al., 1979) would have been sufficient to make blocking a more interesting variable. In addition, the short interval between the stimuli in a pair $(350 \mathrm{msec})$ may have discouraged subjects from assigning covert labels to the precursors.

Single-formant contrast is predicted by Crowder's $(1978,1981,1982,1983)$ theory. The hypothesis is that vowels are represented in auditory memory in some form similar to a sound spectrogram. When two tokens are together in auditory memory, they show frequency-specific lateral inhibition; that is, where the two share formant energy, they mutually weaken each other's representation. This process is shown schematically in Figure 2 for an $/ a /-/ \mathfrak{a} /$ pair.

The inhibition has no effect on vowel quality when formants match from the two vowels. However, when formants partially overlap, as in the illustration of Figure 2 or in F1 of nearby members of the /i//I/ continuum, the intersection region will be inhibited in both. This leaves the most extreme regions of the intersecting formants intact, giving them more extreme formant center frequencies after inhibition than they had before. The absence of contrast from /I/ tokens is, of course, as baffling to this model as it is to most others. At this point, we decided to replicate our experiment with another vowel continuum, in order to see whether these findings had any generality.

PREDICTION OF PHONETIC CONTRAST IN VOWELS FROM FREOUENCY-SPECIFIC LATERAL INHIBITION IN AUDITORY MEMORY

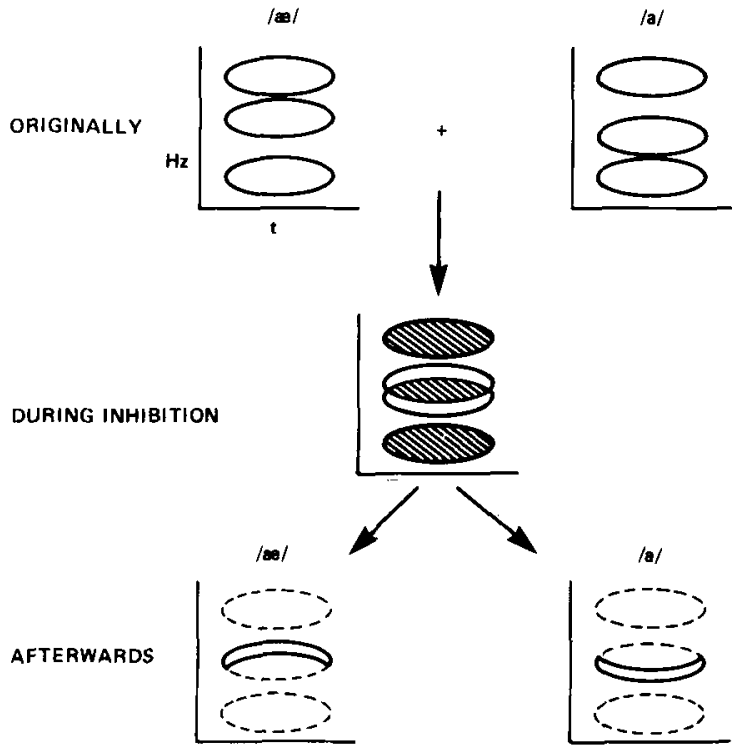

Figure 2. An illustration of how frequency-specific lateral inhibition could produce phonetic contrast in vowels. See text for explanation. 


\section{EXPERIMENT 2}

The second experiment used vowels from the $/ \varepsilon /$ $/ \mathfrak{a} /$ continuum and dropped the comparison of randomized and blocked conditions, using blocked presentation only. Otherwise, it was nearly identical to Experiment 1 . The purpose was simply to generalize to different subjects and different vowels the occurrence of single-formant contrast.

\section{Method}

Subjects. The subjects were 30 Yale undergraduates, participating in return for pay.

Stimuli. Another seven-vowel continuum was prepared on the Haskins Laboratories software serial synthesizer. (No parallel synthesizer was available to us at the time.) The vowels were designed to range perceptually from $/ \varepsilon /$ to $/ \mathfrak{x} /$ and varied, this time, both in F1 and F2 (respectively, from 530 to $660 \mathrm{~Hz}$ and from 1840 to $1720 \mathrm{~Hz}$ ); F3 was fixed at $2480 \mathrm{~Hz}$. The response alternatives on the extremes of the 5-point rating scale were the words BET and BAT. Low-pass filtering (cutoff frequency $=800 \mathrm{~Hz}$, rolloff $=48 \mathrm{~dB} /$ octave) was used in order to produce endpoint tokens of $/ \varepsilon /$ and $/ a e /$ with $F 2$ and F3 deleted. These altered versions of $/ \varepsilon /$ and $/ \mathfrak{a} /$ sounded to us unambiguously like $/ \mathbb{N}$ and $/ \mathrm{J}$, respectively. The same hiss was used as in Experiment 1. The items were all $260 \mathrm{msec}$ long, the ISI was $.35 \mathrm{sec}$, and the delay between trials was set at $3.5 \mathrm{sec}$. In all other procedural details, this experiment was identical to the blocked condition of the previous one.

\section{Results}

The results are shown in Figure 3, which is organized identically to Figure 1 . Evident in the figure are several findings: (1) Contrast from the $/ \varepsilon /$ direction on the vowel continuum occurred both for the full-vowel precursors and for the single formants; (2) this contrast was larger for the full vowels, however, than for the single formants; (3) there was no trace of contrast from the / $\mathbf{x} /$ direction; and (4) there was again a tendency for contrast to "bulge" in the most phonetically ambiguous region of the target items when the full vowels were precursors, but not when the single formants were used.

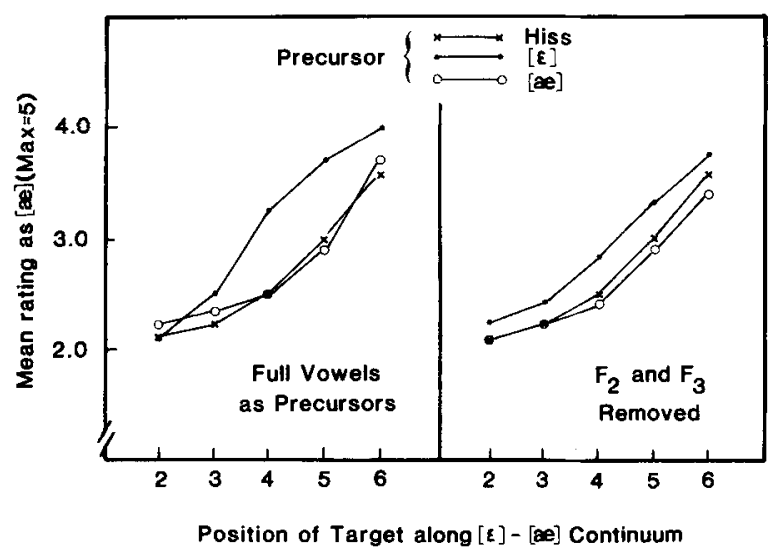

Figure 3. The same as Figure 1 except the data are for an $/ \varepsilon-$ ae/ continuum (Experiment 2).
These observations were confirmed by several analyses of variance on mean ratings (toward $/ \mathfrak{a} /$ ). In the first of these, all five precursor conditions were crossed with the five target vowels. Both main effects and the interaction were statistically significant [for conditions, $F(4,116)=13.32, p<.01 ;$ for target vowels, $F(4,116)=43.46, p<.01$; and for the interaction, $F(16,464)=4.49, p<.01]$. In the next analysis, the hiss condition was dropped, leaving a $2 \times 2$ design with respect to the precursor (full vowels vs. $F 1$ only $\times$ direction of contrast-from $/ \varepsilon /$ vs. from $/ \mathfrak{a} /)$. The five target vowels were compared in the third factor. There were statistically significant main effects of whether the full or altered vowels were used as precursors $[\mathrm{F}(1,29)=6.33, \mathrm{p}<.01]$, of the direction of contrast $[F(1,29)=47.12, p<.01]$, and of the placement on the continuum of the target $[F(4,116)$ $=43.41]$. Given the uniform results of precursors from the $/ \mathfrak{a} /$ direction, the first of these main effects means that the full vowels produced more contrast than the first formants alone. The reliable interaction between direction of contrast and the five target vowels $[F(4,116)=7.81, p<.01]$ indicates that, with the full and altered vowels combined, the interior (ambiguous) vowels were more affected than those closer to the endpoints were. The three-way interaction between precursor type (full vs. altered), direction of contrast, and vowel was statistically significant here, as in the previous experiment $[F(4,116)$ $=3.96, \mathrm{p}<.01]$. This interaction is the most direct verification of the "bulging" in contrast effects for the full vowels but not for the altered ones.

The main empirical goal of this article is the establishment of single-formant contrast. Therefore, since single-formant contrast was smaller than full-vowel contrast in this experiment, one additional analysis of variance was performed, including only two precursor conditions, the hiss control and the singleformant alteration of $/ \varepsilon /$. In this analysis, the main effect associated with this comparison was reliable at the .01 level of confidence $[F(1,29)=8.68]$. The position of the target vowel was, of course, also a reliable source of variation $[F(4,116)=41.29, p<.01]$, but the interaction was less than 1.00 . Thus, both experiments require the conclusion that single-formant contrast can occur on a vowel continuum even when these precursors do not resemble phonetically the vowels targeted for identification.

\section{GENERAL DISCUSSION}

The two studies are so very consistent in most respects, we should deal with the single major discrepancy first: In Experiment 1, the total amount of contrast was no larger for the full vowels than for the single-formant precursors, but in Experiment 2, contrast was larger for the full vowels. One difference in the target stimuli used in the experiment may be 
critical here. In Experiment 2, there was variation between $/ \varepsilon /$ and $/ \mathfrak{a} /$ in both of the first two formants, whereas in Experiment 1, only the first formant varied along the continuum. Methodologically, this discrepancy appears at first inexcusable but, in fact, was desirable to get "prototype" exemplars of both phonetic categories (see Peterson \& Barney, 1952). The consequence is that in Experiment 2, an ambiguous item on the $/ \varepsilon /-/ \mathfrak{z} /$ continuum was receiving potential contrastive influences from both F1 and F2 in the case of the full precursors but only from F1 in Experiment 1. It will be straightforward to untangle these factors in future research should anyone ever be interested in whether single-formant contrast is numerically equal to regular contrast along continua varying in only one formant.

In both experiments, the full vowels produced markedly more contrast for the more ambiguous tokens than for those from near the endpoints (the "bulge"), whereas the altered, single-formant vowels produced relatively uniform contrast across the entire target continuum. We are intrigued by this result but can offer little guidance in its interpretation. An obvious possibility for the locus of an interpretation is in some phonetic process. The identification targets that receive the most contrastive influence from the full vowels are those that are most ambiguous phonetically. Correspondingly, what distinguishes the full vowels from their single-formant variants is that they carry clear phonetic information about the relevant continuum. Somehow the richness of the phonetic information in the precursor could combine judgmentally with the precarious initial classification of the ambiguous target. But such a simple appeal to judgmental bias will obviously not work, because there is no corresponding selective effect of the putative phonetic process when contrast is measured from the "wrong" direction (that is, from either /I/ or from $/ \mathfrak{a} /$ ).

No contrast was obtained in either study when one continuum endpoint was used, and abundant contrast was obtained when the other was used. What is the principle responsible for these asymmetries? With data on only two continua, we would be foolish to propose a general hypothesis. We are now following several theoretical possibilities experimentally. The main burden of this paper is not the asymmetry in contrast observed here and elsewhere, however. In both experiments, we found unambiguous evidence that single-formant precursors affect ambiguous vowel identification. This much was predicted by Crowder's $(1978,1982,1983)$ theory. There may well be other theories that predict single-formant vowel contrast and so we shall not stress the confirmation of these results for that one particular prediction. More to the point, the sort of theory that assigns contrast in this situation to specific, sensory processes is advanced by single-formant contrast.
If subjects were trying to "balance out" their use of the response categories between their internal naming of the precursor and their explicit rating of the target, the single-formant precursors should have been much less effective.

One caveat needs to be added: Removal of F2 and F3 in Experiment 1 made / $/$ / sound like / $u /$ and /I/ sound like either $/ \mathrm{u} /$ or $/ \mathrm{o} /$. Now $/ \mathrm{i} /$ and $/ \mathrm{u} /$ share the feature of being high vowels whereas $/ \mathrm{I} /$ and $/ \mathrm{O} /$ are both articulated with the tongue in a lower position. If subjects in Experiment 1 heard the altered vowels as an $/ \mathrm{u} /-/ \mathrm{o} /$ contrast (14 of the 25 subjects in the preliminary identification test did) and if they were somehow sensitive to the high-low feature, it is possible that they applied a judgmental bias with respect to that feature. That is, if they heard what they thought was $/ u$ / as the first member of the pair, followed by an ambiguous token between $/ \mathrm{i} /$ and $/ \mathrm{I} /$, they might be biased to pick the lower tongueposition alternative, /I/. A similar argument can be applied to $/ \varepsilon /$ and $/ \mathfrak{e} /$ in Experiment 2, which might engage both the high-low and front-back dimensions. We cannot dismiss this possibility on the basis of the present experiments. However, this sort of process would encounter just as much difficulty with the asymmetry of contrast along the various vowel continua as do other notions. Also, in Experiment 1, in which we have data on identification of the precursors, only about half of the subjects would have been expected to identify the single-formant vowels as an $/ \mathrm{u} /-/ \mathrm{o} /$ contrast, if we believe the $14 / 25$ ratio of the preliminary experiment. Furthermore, this alternative explanation requires considerable abstraction of distinctive vowel features, which would be important if true but remains highly speculative now. And finally, the F1 dimension in vowel space is very highly correlated with tongue height, so that the tongueheight dimension is just another level of discourse in which one can talk about $F 1$ frequency.

\section{REFERENCES}

Crowder, R. G. (1978). Mechanisms of backward masking in the stimulus suffix effect. Psychological Review, 85, 502-524.

Crowder, R. G. (1981). The role of auditory memory in speech perception and discrimination. In T. Myers, J. Laver, \& J. Anderson (Eds.), The cognitive representation of speech. Amsterdam: North Holland.

Crowder, R. G. (1982). Decay of auditory memory in vowel discrimination. Journal of Experimental Psychology: Learning, Memory, and Cognition, 8, 153-162.

Crowder, R. G. (1983). The purity of auditory memory. Philosophical Transactions of the Royal Society, Section B., 302, 251-265.

Delattre, P., Liberman, A. M., Cooper, F. S., \& Gerstman, L. (1952). An experimental study of the acoustic determinants of vowel color; observations on one- and two-formant vowels synthesized from spectrographic patterns. Word, 8, 195-210.

Diehl, R. L., Elman, J. L., \& McCusker, S. B. (1978). Contrast effects in stop consonant identification. Journal of Experimental Psychology: Human Perception and Performance, 4, 599-609. 
Diehl, R. L., Lang, M., \& Parker, E. M. (1980). A further parallel between adaptation and contrast. Journal of Experimental Psychology: Human Perception and Performance, 6, 24-44.

Fry, D. B., Abramson, A. S., Eimas, P. D., \& Liberman, A. M. (1962). The identification and discrimination of synthetic vowels, Language and Speech, 5, 171-189.

Gilchrist, A. (1977). Perceived lightness depends on perceived spatial arrangement. Science, 195, 185-187.

Lindsay, P. H., \& Norman, D. A. (1977). Human information processing (2nd ed.). New York: Academic Press.

Parducci, A. (1974). Contextual effects: A range-frequency analysis. In E. C. Carterette \& M. P. Friedman (Eds.), Handbook of perception (Vol. 2). New York: Academic Press.

Peterson, G. E., \& Barney, H. L. (1952). Control methods used in a study of the identification of vowels. Journal of the Acoustical Society of America, 24, 175-184.
Repp, B. H., Healy, A. F., \& Crowder, R. G. (1979). Categories and context in the perception of isolated, steady-state vowels. Journal of Experimental Psychology: Human Perception and Performance, 5, 129-143.

Roberts, M., \& Summerfield, Q. (1981). Audiovisual presentation demonstrates that selective adaptation in speech perception is purely auditory. Perception \& Psychophysics, 30, 309-314.

Sawusch, J. R., \& JusczYK, P. (1981). Adaptation and contrast in the perception of voicing. Journal of Experimental Psychology: Human Perception and Performance, 1, 408-421.

Sawusch, J. R., Nusbaum, H. C., \& Schwab, E. C. (1980). Contextual effects in vowel perception II: Evidence for two processing mechanisms. Perception \& Psychophysics, 27, 421-434.

(Manuscript received July 29, 1983;

revision accepted for publication December 30,1983.) 